\title{
Diabetes is not associated with greater pain and worse patient-reported outcome measures one year after laminoplasty for cervical spondylotic myelopathy
}

\section{Kosei Nagata}

The University of Tokyo Spine Group (UTSG)

Junya Miyahara

The University of Tokyo

Hideki Nakamoto

The University of Tokyo

Naohiro Kawamura

Japanese Red Cross Medical Center

Yujiro Takeshita

Yokohama Rosai Hospital

Akiro Higashikawa

Kanto Rosai Hospital

Takashi Ono

Japan Community Health-care Organization Tokyo Shinjuku Medical Center

Masayoshi Fukushima

Toranomon Hospital

\section{Rentaro Okazaki}

Saitama Red Cross Hospital

Nobuhiro Hara

Musashino Red Cross Hospital

\section{So Kato}

The University of Tokyo Spine Group (UTSG)

\section{Toru Doi}

The University of Tokyo

\section{Yuki Taniguchi}

The University of Tokyo

Yoshitaka Matsubayashi

The University of Tokyo

\section{Sakae Tanaka}

The University of Tokyo 


\section{Yasushi Oshima ( $\nabla$ yoo-tky@umin.ac.jp )}

The University of Tokyo Spine Group (UTSG)

\section{Research Article}

Keywords: group, pain, proms, laminoplasty, neck

Posted Date: November 2nd, 2021

DOI: https://doi.org/10.21203/rs.3.rs-1025886/v1

License: (c) (1) This work is licensed under a Creative Commons Attribution 4.0 International License. Read Full License 


\section{Abstract}

Although patients with diabetes reportedly have more peripheral neuropathy, the impacts of diabetes on postoperative recovery in pain and patient-reported outcome measures (PROMs) after laminoplasty for cervical spondylotic myelopathy (CSM) is not well characterized. The authors aimed to elucidate the effects of diabetes on neck/arm/hand/leg/foot pain and PROMs after laminoplasty CSM. The authors retrospectively reviewed 339 patients ( 82 with diabetes and 257 without) who underwent laminoplasty between C3 and C7 in 11 hospitals during April 2017-October 2019. Preoperative Numerical Rating Scale (NRS) scores in all five areas, the Short Form-12 Mental Component Summary, Euro quality of life 5dimension, Neck Disability Index, and the Core Outcome Measures Index-Neck) were comparable between the groups. The between-group differences were also not significant in NRS scores and PROMs one after surgery. The change score of NRS hand pain was larger in the diabetic group than the nondiabetic group. The diabetic group showed worse preoperative score but greater improvement in the Short Form-12 Physical Component Summary than the nondiabetic group, following comparable score one year after surgery. These data indicated that the preoperative presence of diabetes, at least, did not adversely affect pain or PROMs one year after laminoplasty for CSM.

\section{Introduction}

Cervical spondylotic myelopathy (CSM) is a common and increasingly observed degenerative disorder causing spinal cord compression and neurological deterioration. ${ }^{1}$ Surgical decompression via laminoplasty is the standard treatment for CSM and can prevent the progression of neurological deficits as well as improve neurological function and quality of life, ${ }^{2}$ which should be evaluated using patientreported outcome measures (PROMs).

T Diabetic neuropathy following diabetes mellitus can profoundly impair the quality of life. Diabetes mellitus is a chronic systemic disease characterized by multiple neurologic sequelae mainly associated with diabetic neuropathy, chronic peripheral pain due to microvascular changes, and irreversible nerve damage. ${ }^{3-5}$ The reported prevalence of painful diabetic peripheral neuropathy shows wide variability (range, $3 \%-65 \%$ ) owing to differences in sampling methods and diagnostic criteria. ${ }^{3-9}$ It is a well-known fact that patients with diabetic neuropathy have sensory disturbances in the extremities and typically show stocking-and-glove patterns of sensory deficit. ${ }^{10}$ Compromised vascularity and secondary peripheral neuropathy may affect the recovery of nerve roots even after surgical decompression. ${ }^{11}$

Only a few large-scale studies have investigated the effects of diabetes on laminoplasty outcomes, and their results were controversial. ${ }^{12-14}$ These studies included patients undergoing anterior cervical spine surgeries ${ }^{12}$ and did not investigate PROMs ${ }^{13}$ or peripheral pain. ${ }^{14}$ Therefore, a larger study based on prospectively collected data is required to evaluate the effects of diabetes on PROMs and pain in the neck, arm, hand, leg, and foot areas after cervical laminoplasty surgery. In the present multicenter study 
investigating the surgical outcomes of laminoplasty for CSM, the authors aimed to investigate the impact of diabetes on pain as well as PROMs after laminoplasty for CSM with an adequately large sample size.

\section{Materials And Methods}

\section{Ethics}

A prospective spine surgery registry was started at eight institutions in the greater Tokyo metropolitan area, after obtaining approval from the Clinical Research Support Center of the University of Tokyo Hospital (10335-(3)) and the institutional review boards of all participating hospitals i.e., The University of Tokyo Hospital, Japanese Red Cross Medical Center, Yokohama Rosai Hospital, Kanto Rosai Hospital, Japan Community Health-care Organization Tokyo Shinjuku Medical Center, Toranomon Hospital, Japanese Red Cross Saitama Hospital, and Japanese Red Cross Musashino Hospital. The present study was carried out in accordance with the relevant guidelines and regulations/ethical principles of the Declaration of Helsinki. The authors have obtained informed consent form with opt-out method from patients.

\section{Patients}

The authors evaluated a consecutive cohort of patients who were clinically and radiographically diagnosed with CSM and underwent posterior cervical decompression at 11 different institutions between April 2017 and October 2019. The study's inclusion criteria were as follows: symptomatic CSM (at least one clinical sign of myelopathy), surgical level between C3 and C7, evidence of cervical spinal cord compression on magnetic resonance imaging or cervical myelogram-computed tomography, and no previous cervical spine surgery. The study's exclusion criteria were as follows: diagnosis of posterior longitudinal ligament ossification, spinal tumors, trauma, or infectious diseases; aged younger than 18 years; emergency surgery; surgery involving the thoracic spine; and fixation surgery.

\section{Patient data collection}

Data on the clinical characteristics of the patients, including age, sex, body mass index, current smoking history, American Society of Anesthesiologists (ASA) classification, and medical history, were retrospectively collected. Preoperative laboratory data were assessed. Patients with a fasting blood glucose level of $126 \mathrm{mg} / \mathrm{dL}$ and glycated hemoglobin (HbA1c) levels of $6.5 \%$ or those previously

diagnosed with diabetes by a diabetes specialist were considered to have diabetes. ${ }^{15}$ Diabetes specialists at each hospital were able to sufficiently control the blood sugar levels of patients diagnosed with diabetes throughout the immediate perioperative period. Surgical factors, including operative time and estimated blood loss, were registered. Surgeons in charge were asked to report all intraoperative complications, including nerve root damage and dural tear, and postoperative complications within 30 days after surgery.

\section{Patient-reported outcome measures}


At baseline and one year after surgery, all patients were asked to answer a booklet of questionnaires, including the Japanese version of the (1) Numerical Rating Scale (NRS), (2) the Short Form-12 Physical Component Summary (PCS), (3) Mental Component Summary (MCS), (4) Euro quality of life 5-

dimmension (EQ-5D)-3L to assess health-related quality of life ${ }^{16}(5)$ Neck Disability Index (NDI) to assess pain-associated disability, and (6) Core Outcome Measures Index (COMI)-Neck. ${ }^{17}$ The NRS measures the intensity of pain over the preceding 4 weeks; the scores range from 0 (no pain at all) to 10 (worst pain imaginable). To evaluate the effect of CSM on both neck pain and radiculopathy, the authors analyzed the following five NRS domains; neck, arm, hand, leg, and foot pain. Each corresponding body part had been discussed in previous reports. ${ }^{18}$ To determine treatment satisfaction, a 7-point Likert scale was used, wherein, the patients were asked to answer whether they were satisfied with the treatment, with possible answers of "very satisfied," "satisfied," "somewhat satisfied," "neither," "dissatisfied," "somewhat dissatisfied," and "very dissatisfied."17

\section{Statistical analysis}

Baseline demographic and clinical characteristics in the diabetic and nondiabetic groups were compared using the Chi-square test for categorical variables and Student's $t$-test for continuous variables. The Student's $t$-test was used to examine intergroup differences with respect to pre- and postoperative NRS, PCS, MCS, EQ-5D, NDI, and COMI-Neck scores. For further evaluation of the difference in each outcome score, the authors calculated the adjusted $p$ values by inverse probability weighting method after calculating propensity scores based on seven variables (age, sex, BMI, smoking status, ASA class, operative time, and estimated blood loss) as per a previous report. ${ }^{12,19}$ All data analyses were performed using SPSS version 21.0 statistical software (SPSS, Inc., Chicago, IL, USA).

The sample size for this study was calculated using G*Power version 3.1. With an approximately $20 \%$ $-25 \%$ prevalence of diabetes as reported in a previous prospectively collected database, ${ }^{13,20}$ a total sample size of at least 200 patients was required with a power of $>0.8$, significance level of $P<0.05$, and effect size of $<0.2$.

\section{Results}

Among the 339 patients who satisfied the inclusion and exclusion criteria, 82 (24.2\%) and 257 (75.8\%) were grouped into the diabetic and nondiabetic groups, respectively (Figure 1). No significant intergroup differences were observed with respect to age (mean 70.1. vs. 71.0 years, $P=0.575$ ), male/female ratio (58 men:24 women vs. 175 men:82 women, $P=0.653)$, and current smoking status $(12.2 \%$ smokers vs. $11.1 \%$ nonsmokers, $\mathrm{P}=0.454)$ (Table 1$)$. The diabetic group had a higher $\mathrm{BMI}\left(25.2 \mathrm{vs} .23 .7 \mathrm{~kg} / \mathrm{m}^{2}, \mathrm{P}=\right.$ $0.012)$ and included a higher percentage of patient with an ASA classification of $\geq 3(19.5 \%$ vs. $8.0 \%, P<$ $0.001)$, hypertension ( $58.6 \%$ vs. $41.1 \%, P=0.022)$, and hyperlipidemia $(26.4 \%$ vs. $11.1 \%, P=0.006)$ than the nondiabetic group. No significant intergroup differences were observed with respect to hemodialysis, rheumatoid arthritis, and past history of stroke. The diabetic group had a significantly higher mean HbA1c level (7.0\% vs. $5.8 \%, P<0.001)$, fasting blood sugar (161.3 vs. $112.2 \mathrm{mg} / \mathrm{dL}, \mathrm{P}<0.001$ ), and 
creatinine (1.4 vs. $1.0 \mathrm{mg} / \mathrm{dL}, \mathrm{P}=0.009)$ than the nondiabetic group. The diabetic group had a lower mean value of total cholesterol than the nondiabetic group (183.1 vs. $200.9 \mathrm{mg} / \mathrm{dL}, \mathrm{P}=0.001)$. Although the diabetic group had a lower value of mean low density lipoprotein cholesterol than the nondiabetic group, the difference was not statistically significant (97.4 vs. $110.4 \mathrm{mg} / \mathrm{dL}, \mathrm{P}=0.059$ ).

Table 1

Demographic and Patient Characteristics

\begin{tabular}{|c|c|c|c|}
\hline & Diabetic Group & Nondiabetic Group & \\
\hline & $(\mathrm{N}=82)$ & $(N=257)$ & $P$ value \\
\hline $\mathrm{Age}^{\star}(\mathrm{yr})$ & $70.1 \pm 11.3$ & $71.0 \pm 11.0$ & 0.575 \\
\hline Male/Female $†$ & $58 / 24$ & $175 / 82$ & 0.653 \\
\hline $\mathrm{BMI}^{\star}(\mathrm{kg} / \mathrm{m} 2)$ & $25.2 \pm 4.9$ & $23.7 \pm 3.6$ & 0.012 \\
\hline Current Smokert (\%) & 12.2 & 11.1 & 0.454 \\
\hline ASA classification $\geq 3+(\%)$ & 19.5 & 8.0 & 0.033 \\
\hline Hypertension† (\%) & 58.6 & 41.1 & 0.022 \\
\hline Hyperlipidemia† (\%) & 26.4 & 11.1 & 0.006 \\
\hline Hemodialysist (\%) & 6.1 & 1.9 & 0.053 \\
\hline Rheumatoid Arthritist (\%) & 1.2 & 1.9 & 0.664 \\
\hline Past history of stroket (\%) & 9.8 & 4.4 & 0.115 \\
\hline $\mathrm{HbA} 1 \mathrm{c}^{*}$ & $7.0 \pm 0.8$ & $5.8 \pm 0.5$ & $<0.001$ \\
\hline Fasting blood sugar* & $161.3 \pm 52.2$ & $112.2 \pm 32.1$ & $<0.001$ \\
\hline Creatinine* & $1.4 \pm 1.9$ & $1.0 \pm 1.0$ & 0.009 \\
\hline Total Cholesterol* & $183.1 \pm 44.4$ & $200.9 \pm 34.0$ & 0.007 \\
\hline Low Density Lipoprotein cholesterol* & $97.4 \pm 34.7$ & $110.4 \pm 29.0$ & 0.059 \\
\hline
\end{tabular}

No significant intergroup differences were observed with respect to operative time (139.7 vs. $136.2 \mathrm{~min}, \mathrm{P}$ $=0.590$ ) and estimated blood loss (102.0 vs. $98.4 \mathrm{~mL}, \mathrm{P}=0.855$ ) (Table 2). No significant intergroup differences were observed in complications during surgery and within 30 days after surgery. 
Table 2

Comparison of Surgical Factors for Patients with Diabetes and Those without Diabetes

\begin{tabular}{|llll|}
\hline & $\begin{array}{l}\text { Diabetic Group } \\
(\mathbf{N}=\mathbf{8 2})\end{array}$ & $\begin{array}{l}\text { Nondiabetic Group } \\
\mathbf{( N = 2 5 7 )}\end{array}$ & P value \\
\hline Operation time* $(\mathrm{min})$ & $139.7 \pm 45.9$ & $136.2 \pm 51.5$ & 0.590 \\
\hline Estimated blood loss* $(\mathrm{ml})$ & $102.0 \pm 162.5$ & $98.4 \pm 153.7$ & 0.855 \\
\hline Nerve root damage $(\%)$ & 0 & 0 & NA \\
\hline Dural tear $(\%)$ & 1.2 & 2.3 & 0.863 \\
\hline Surgical site infection $(\%)$ & 3.7 & 2.7 & 0.952 \\
\hline Urinary tract infection $(\%)$ & 2.4 & 0.8 & 0.532 \\
\hline Respiratory tract infection $(\%)$ & 2.4 & 0.4 & 0.294 \\
\hline $\begin{array}{l}* \\
\text { percentage in each group. }\end{array}$ & & & \\
\hline
\end{tabular}

The absolute changes in NRS scores are shown in Table 3. Pre- and postoperative NRS scores in neck/arm/leg/foot were comparable between the two groups $(P>0.05)$. There were no significant differences in the change NRS scores in neck/arm/leg/foot with or without adjustment by propensity score. Although pre- and postoperative NRS hand pain scores showed no significant difference between the two groups, the change score of NRS hand pain was larger in the diabetic group than the nondiabetic group (1.2 vs. 0.3, $P=0.034$, Adjusted $P=0.017$ ). 
Table 3

Comparison of Preoperative and Postoperative Numeric Rating Scales for Patients with Diabetes and Those without Diabetes

\begin{tabular}{|llllll|}
\hline NRSs & Outcome & $\begin{array}{l}\text { Diabetic } \\
\text { Group }\end{array}$ & $\begin{array}{l}\text { Nondiabetic } \\
\text { Group }\end{array}$ & $\begin{array}{l}\text { P } \\
\text { vlaue }\end{array}$ & $\begin{array}{l}\text { Adjusted P } \\
\text { value }\end{array}$ \\
\hline NRS Neck pain & Preoperative & $2.4 \pm 2.9$ & $2.8 \pm 2.8$ & 0.290 & 0.439 \\
& Postoperative & $2.1 \pm 2.3$ & $2.0 \pm 2.3$ & 0.909 & 0.986 \\
\hline & Change & $0.3 \pm 3.2$ & $0.8 \pm 2.9$ & 0.169 & 0.334 \\
\hline NRS Arm pain & Preoperative & $3.1 \pm 3.3$ & $3.0 \pm 3.0$ & 0.797 & 0.649 \\
\hline & Postoperative & $1.9 \pm 2.6$ & $2.3 \pm 2.8$ & 0.253 & 0.208 \\
\hline NRS Hand & Change & $1.3 \pm 3.6$ & $0.6 \pm 3.2$ & 0.166 & 0.146 \\
\hline & Preoperative & $3.3 \pm 3.3$ & $3.0 \pm 3.1$ & 0.478 & 0.296 \\
\hline & Postoperative & $2.0 \pm 2.7$ & $2.6 \pm 2.9$ & 0.113 & 0.141 \\
\hline NRS Leg pain & Change & $1.2 \pm 3.2$ & $0.3 \pm 3.3$ & 0.034 & 0.017 \\
\hline & Preoperative & $2.6 \pm 3.1$ & $2.9 \pm 3.0$ & 0.505 & 0.565 \\
\hline & Postoperative & $2.5 \pm 2.8$ & $2.5 \pm 2.9$ & 0.968 & 0.738 \\
\hline & Change & $0.1 \pm 3.3$ & $0.3 \pm 3.4$ & 0.918 & 0.932 \\
\hline NRS, Numeric Rating Scale & & $1.9 \pm 2.7$ & 0.701 & 0.635 \\
\hline & Preoperative & $1.8 \pm 2.9$ & $1.7 \pm 2.7$ & 0.809 & 0.587 \\
\hline & Postoperative & $1.8 \pm 2.5$ & $0.1 \pm 2.7$ & 0.189 & 0.628 \\
\hline & Change & $0.0 \pm 2.9$ & & & \\
\hline & & & & \\
\hline
\end{tabular}

The trends in PROMs are shown in Table 4. The diabetic group had a lower preoperative PCS score than the nondiabetic group with or without adjustment (22.0 vs. 28.9, $P=0.001$, Adjusted $P=0.028)$, and no significant intergroup differences were observed in postoperative PCS scores $(30.4$ vs. $32.7, P=0.324)$. Hence, the change score in PCS was larger in the diabetic group than the nondiabetic group, but the difference was not significant after the adjustment by the propensity score (8.4 vs. 3.7, $P=0.039$, Adjusted $P=0.151$ ). No significant intergroup differences were observed in pre- and postoperative MCS, EQ-5D, NDI, and COMI-Neck scores. 
Table 4

Comparison of Preoperative and Postoperative Patient Reported Outcome Measures for Patients with Diabetes and Those without Diabetes

\begin{tabular}{|c|c|c|c|c|c|}
\hline PROMs & Outcome & Diabetic Group & Nondiabetic Group & P vlaue & Adjusted P value \\
\hline \multirow[t]{3}{*}{ SF-12 PCS } & Preoperative & $22.0 \pm 15.5$ & $28.9 \pm 17.0$ & 0.001 & 0.028 \\
\hline & Postoperative & $30.4 \pm 16.7$ & $32.7 \pm 16.2$ & 0.324 & 0.091 \\
\hline & Change & $8.4 \pm 16.9$ & $3.7 \pm 15.3$ & 0.039 & 0.151 \\
\hline \multirow[t]{3}{*}{ SF-12 MCS } & Preoperative & $51.6 \pm 11.7$ & $48.8 \pm 10.7$ & 0.072 & 0.165 \\
\hline & Postoperative & $53.4 \pm 11.1$ & $52.2 \pm 9.5$ & 0.336 & 0.352 \\
\hline & Change & $2.1 \pm 12.2$ & $3.3 \pm 11.5$ & 0.185 & 0.396 \\
\hline \multirow[t]{3}{*}{ EQ-5D } & Preoperative & $0.54 \pm 0.27$ & $0.56 \pm 0.22$ & 0.464 & 0.927 \\
\hline & Postoperative & $0.63 \pm 0.16$ & $0.65 \pm 0.21$ & 0.536 & 0.727 \\
\hline & Change & $0.09 \pm 0.25$ & $0.08 \pm 0.21$ & 0.941 & 0.834 \\
\hline \multirow[t]{3}{*}{ NDI } & Preoperative & $37.7 \pm 19.7$ & $38.1 \pm 20.2$ & 0.871 & 0.637 \\
\hline & Postoperative & $27.0 \pm 17.5$ & $27.4 \pm 18.4$ & 0.891 & 0.886 \\
\hline & Change & $9.3 \pm 18.6$ & $10.6 \pm 20.4$ & 0.563 & 0.326 \\
\hline \multirow[t]{3}{*}{ COMI-Neck } & Preoperative & $5.5 \pm 2.2$ & $5.8 \pm 2.1$ & 0.316 & 0.189 \\
\hline & Postoperative & $3.5 \pm 2.2$ & $3.9 \pm 2.5$ & 0.248 & 0.257 \\
\hline & Change & $1.9 \pm 2.3$ & $2.0 \pm 2.7$ & 0.692 & 0.493 \\
\hline
\end{tabular}

Table 5 summarizes the answers to questions on satisfaction. The diabetic group tended to have a higher proportion of patients answering "very satisfied" or "satisfied" than the nondiabetic group, although the difference was not significant ( $58.5 \%$ vs. $47.5 \%, \mathrm{P}=0.081)$. The diabetic group had a higher total percentage of patients who answered "very satisfied," "satisfied," or "somewhat satisfied" than the nondiabetic group $(74.4 \%$ vs. $64.6 \%, P=0.001)$. 
Table 5

Comparison of Satisfaction between the Diabetic and Nondiabetic group.

\begin{tabular}{|lll|}
\hline & $\begin{array}{l}\text { Diabetic Group } \\
\mathbf{( N = 8 2 )}\end{array}$ & $\begin{array}{l}\text { Nondiabetic Group } \\
\mathbf{( N = 2 5 7 )}\end{array}$ \\
\hline Very satisfied -n (\%) & $15(18.3)$ & $50(19.5)$ \\
\hline Satisfied -n (\%) & $33(40.2)$ & $72(28.0)$ \\
\hline Somewhat satisfied -n (\%) & $13(15.9)$ & $44(17.1)$ \\
\hline Neither -n (\%) & $11(13.4)$ & $59(23.0)$ \\
\hline Dissatisfied -n (\%) & $3(3.7)$ & $9(3.5)$ \\
\hline Somewhat dissatisfied -n (\%) & $4(4.9)$ & $13(5.1)$ \\
\hline Very dissatisfied -n (\%) & $3(3.7)$ & $10(3.9)$ \\
\hline
\end{tabular}

\section{Discussion}

To the best of our knowledge, this has been the largest study that investigated the effects of diabetes on multiple NRS scores with PROMs following elective laminoplasty for CSM. In the present study, pre-and postoperative NRS scores for neck/arm/hand/leg/foot pain were comparable between the two groups. The diabetic group showed greater improvement in NRS hand scores than the nondiabetic group. No significant intergroup differences in pre- and postoperative MCS, EQ-5D, NDI, and COMI-Neck scores were noted. Although the diabetic group had lower preoperative PCS scores than the nondiabetic group, the postoperative PCS scores of the two groups were comparable. Moreover, patients in the diabetic group tended to be satisfied with the surgical results. Collectively, these data indicated that the presence of diabetes did not, at least, adversely affect surgical outcomes of laminoplasty for CSM.

The present study showed that the diabetic group had higher prevalence rates of ASA classification $\geq 3$, consistent with the findings of a previous study. ${ }^{13}$ The diabetic group also had more patients undergoing hemodialysis and higher mean creatinine levels than the nondiabetic group. Although the diabetic group tended to have more risk factors, the current study revealed no intergroup differences with respect to perioperative complications. Compared with the nondiabetic group, the diabetic group had a higher proportion of patients diagnosed with hyperlipidemia but lower mean values of total cholesterol and low density lipoprotein cholesterol. These data may reflect good medication control in the diabetic group in the present study. The mean and maximum $\mathrm{HbA} 1 \mathrm{c}$ level was $7.0 \%$ and $8.9 \%$, respectively, in the diabetic group. This relatively strict glycemic control enforced by diabetes specialists before and after laminoplasty surgery may have contributed to the lack of intergroup differences not only in complications but also in the non-inferiority in pain control. Machino et al. argued that patients with diabetes would be more likely to exhibit a reasonable recovery after cervical decompression with appropriate perioperative 
blood glucose control. ${ }^{10}$ Under the appropriate glycemic control, the effect of diabetic neuropathy on pain recovery can be relieved especially in peripheral area; hand.

Only a few large-scale reports have investigated the effects of diabetes on laminoplasty using PROMs. ${ }^{12}$ $\mathrm{n}$ the present study, the diabetic and nondiabetic groups showed similar pre- and postoperative MCS/EQ5D/NDI/COMI-Neck scores, except for PCS. Diabetes has been reported as a risk factor for neck and back pain. ${ }^{21,22}$ Armaghani et al. argued that patients with diabetes showed minor improvements in the NDI or Oswestry Disability Index and EQ-5D scores compared with patients without diabetes. ${ }^{12}$ They analyzed patients undergoing back surgery and those undergoing neck surgery together ${ }^{12}$ and thereby making interpretation difficult. Alternatively, Arnold et al. found that patients with diabetes did not differ from those without diabetes in terms of pre- and postoperative $\mathrm{NDI}^{14}$ and Nori et al. argued that diabetes did not negatively affect neck pain after posterior cervical decompression. ${ }^{13}$ The present study was consistent with these reports. The effects of diabetes may differ between patient with back and neck pain. Given that diabetes impairs bone fusion, the effects of diabetes can be greater following posterior lumbar interbody fusion surgeries, ${ }^{23}$ which contributes to mechanical instability and the occurrence of back pain. ${ }^{13}$ In contrast, considering that cervical laminoplasty is not performed for the purpose of bone fusion, the effects of diabetes can be minor. These mechanisms can explain why no significant differences were observed for neck pain-associated outcome measures, including NRS neck pain, EQ-5D, $\mathrm{NDI}$, and multidimensional COMI-Neck, in the current study.

In the present study, the diabetic group showed lower preoperative PCS scores than the nondiabetic group; however, the two groups had comparable postoperative PCS scores. This finding differed from that reported by Arnold et al ${ }^{4}$; they reported no significant intergroup differences in preoperative PCS, but patients with diabetes experienced significantly lesser improvement than those without diabetes. ${ }^{14}$ Our cohort had an average age of 70.8 years, while Arnold's cohort had an average age of 56.4 years. ${ }^{14}$ The relatively older our cohort with low preoperative PCS scores may have affected the difference. Lower leg strength and proprioception can be associated with low preoperative PCS scores ${ }^{19}$ and greater risk of slower walking speed, ${ }^{24}$ especially in the elderly. ${ }^{25}$ The slower walking speed due to diabetic neuropathy could mimic walking impairment due to CSM, resulting in early surgical intervention in the diabetic group. Early surgical intervention is an important factor for good clinical outcomes ${ }^{26}$ and can explain why the diabetic group in the present study had comparable postoperative PCS scores, despite having lower preoperative PCS scores. To prove this hypothesis, further large size studies linking duration of CSM symptoms and NRS/PROMs are warranted.

The authors believe that this study adds to the contemporary knowledge related to the effect of diabetes on patients undergoing cervical posterior decompression surgery. However, some limitations of our study should be considered when interpreting our results. First, the current study did not analyze detailed information for diabetes except for preoperative $\mathrm{HbA1c}$ level. We were unable to collect detailed information on diabetes, including duration, medication history, or trend of HbA1c. Second, the follow-up duration was one year, not two year after surgery, although PROMs assessed at one year adequately 
predict long-term (24-month) outcomes. ${ }^{27}$ Third, double door or open door laminoplasty was determined by the surgeon in charge at each participating hospital due to the multicenter study design. In addition, relatively elder cohorts may undergo laminoplasty for the purpose myelopathy rather than pain caused by radiculopathy and the effect of laminoplasty on peripheral may be limited.

\section{Conclusions}

The results of the present multicenter prospective cohort study showed that diabetes did not adversely affect improvement in pain and PROMs one year after laminoplasty for CSM, although the diabetic group had lower preoperative PCS scores than the nondiabetic group. Under the strict glycemic control before and after surgery, surgeons can use this information when counseling patients who have CSM with diabetes about the expected outcomes of laminoplasty.

\section{Declarations}

\section{Author contributions}

$\mathrm{KN}$ and $\mathrm{YO}$ conceived the study. JM, HN and SK analyzed the data. TD, YT, and YM discussed the results. $\mathrm{NK}, \mathrm{YT}, \mathrm{AH}, \mathrm{TO}, \mathrm{MF}, \mathrm{RO}$, and $\mathrm{NH}$ contributed data collection and result interpretation. KN wrote the initial draft. YO and ST revised the manuscript. All authors reviewed the manuscript.

\section{Additional information}

None.

The manuscript submitted does not contain information about medical device(s)/drug(s).

No funds were received in support of this work. No benefits in any form have been or will be received from a commercial party related directly or indirectly to the subject of this manuscript. The study approval was given by the institutional review board of the Clinical Research Support Center of the University of Tokyo Hospital.

\section{References}

1 Fehlings, M. G., Tetreault, L. A., Wilson, J. R. \& Skelly, A. C. Cervical spondylotic myelopathy: current state of the art and future directions. Spine (Phila Pa 1976) 38, S1-8, doi:10.1097/BRS.0b013e3182a7e9e0 (2013).

2 Fehlings, M. G.et al. Efficacy and safety of surgical decompression in patients with cervical spondylotic myelopathy: results of the AOSpine North America prospective multi-center study. J Bone Joint Surg Am 95, 1651-1658, doi:10.2106/JBJS.L.00589 (2013). 
3 Vinik, A. I. CLINICAL PRACTICE. Diabetic Sensory and Motor Neuropathy. N Engl J Med 374, 1455-1464, doi:10.1056/NEJMcp1503948 (2016).

4 Ziegler D, R. W., Meisinger C. Prevalence and risk factors of neuropathic pain in survivors of myocardial infarction with pre-diabetes and diabetes. The KORA Myocardial Infarction Registry. Eur J Pain 13, 582587 (2009).

5 Erbas T, E. M., Yucel A. Prevalence of peripheral neuropathy and painful peripheral neuropathy in Turkish diabetic patients. J Clin Neurophysiol 28, 51-55 (2011).

6 Abbott CA, M. R., van Ross ER. Prevalence and characteristics of painful diabetic neuropathy in a large communitybased diabetic population in the U.K. Diabetes Care 34, 2220-2224 (2011).

7 Kim SS, W. J., Kwon HS. Prevalence and clinical implications of painful diabetic peripheral neuropathy in type 2 diabetes: results from a nationwide hospital-based study of diabetic neuropathy in Korea. Diabetes Res Clin Pract 103, 522-529 (2014).

8 Alleman CJ, W. K., Hensen M. Humanistic and economic burden of painful diabetic peripheral neuropathy in Europe: a review of the literature. Diabetes Res Clin Pract 109, 215-225 (2015).

9 Bouhassira D, L. M., Hartemann A. Chronic pain with neuropathic characteristics in diabetic patients: a French crosssectional study. PLoS One 8, e74195 (2013).

10 Machino, M.et al. Risk factors for poor outcome of cervical laminoplasty for cervical spondylotic myelopathy in patients with diabetes. J Bone Joint Surg Am 96, 2049-2055, doi:10.2106/JBJS.N.00064 (2014).

11 Freedman, M. K.et al. The impact of diabetes on the outcomes of surgical and nonsurgical treatment of patients in the spine patient outcomes research trial. Spine (Phila Pa 1976) 36, 290-307, doi:10.1097/BRS.0b013e3181ef9d8c (2011).

12 Armaghani, S. J., Archer, K. R., Rolfe, R., Demaio, D. N. \& Devin, C. J. Diabetes Is Related to Worse Patient-Reported Outcomes at Two Years Following Spine Surgery. J Bone Joint Surg Am 98, 15-22, doi:10.2106/JBJS.0.00297 (2016).

13 Nori, S.et al. Diabetes Does Not Adversely Affect Neurological Recovery and Reduction of Neck Pain After Posterior Decompression Surgery for Cervical Spondylotic Myelopathy: Results From a Retrospective Multicenter Study of 675 Patients. Spine (Phila Pa 1976) 46, 433-439, doi:10.1097/BRS.0000000000003817 (2021).

14 Arnold, P. M.et al. Mild diabetes is not a contraindication for surgical decompression in cervical spondylotic myelopathy: results of the AOSpine North America multicenter prospective study (CSM). The Spine Journal 14, 65-72, doi:10.1016/j.spinee.2013.06.016 (2014). 
15 Committee of the Japan Diabetes Society on the Diagnostic Criteria of Diabetes, M.et al. Report of the committee on the classification and diagnostic criteria of diabetes mellitus. J Diabetes Investig 1, 212228, doi:10.1111/j.2040-1124.2010.00074.x (2010).

16 Tsuchiya A, I. S., Ikegami N, Nishimura S, Sakai I, Fukuda \& T, H. C., Hisashige A, Tamura M. Estimating an EQ-5D population value set: the case of Japan. Health Econ 11 (2002).

17 Oshima, Y.et al. Validity of the Japanese core outcome measures index (COMI)-neck for cervical spine surgery: a prospective cohort study. Eur Spine J 30, 402-409, doi:10.1007/s00586-020-06657-4 (2021).

18 Oshima, Y.et al. Mental State Can Influence the Degree of Postoperative Axial Neck Pain Following Cervical Laminoplasty. Global Spine J 9, 292-297, doi:10.1177/2192568218793861 (2019).

19 Nagata, K.et al. Diabetes is associated with greater leg pain and worse patient-reported outcomes at 1 year after lumbar spine surgery. Scientific Reports 11, doi:10.1038/s41598-021-87615-y (2021).

20 Machino, M.et al. Characteristics of Residual Symptoms After Laminoplasty in Diabetic Patients With Cervical Spondylotic Myelopathy: A Prospective Cohort Study. Spine (Phila Pa 1976) 42, E708-E715, doi:10.1097/BRS.0000000000001947 (2017).

21 Pai, L. W.et al. Musculoskeletal pain in people with and without type 2 diabetes in Taiwan: a population-based, retrospective cohort study. BMC Musculoskelet Disord 16, 364, doi:10.1186/s12891015-0819-4 (2015).

22 Jimenez-Garcia, R.et al. Is there an association between diabetes and neck pain and lower back pain? Results of a population-based study. J Pain Res 11, 1005-1015, doi:10.2147/JPR.S158877 (2018).

23 Henderson, S., Ibe, I., Cahill, S., Chung, Y. H. \& Lee, F. Y. Bone Quality and Fracture-Healing in Type-1 and Type-2 Diabetes Mellitus. J Bone Joint Surg Am 101, 1399-1410, doi:10.2106/JBJS.18.01297 (2019).

24 Cimbiz A, C. O. Evaluation of balance and physical fi tness in diabetic neuropathic patients. $J$ Diab Complications 19, 160-164 (2005).

25 Volpato, S.et al. Role of Muscle Mass and Muscle Quality in the Association Between Diabetes and Gait Speed. Diabetes Care 35, 1672-1679, doi:10.2337/dc11-2202 (2012).

26 Machino, M.et al. Risk Factors for Poor Outcome of Cervical Laminoplasty: Multivariate Analysis in 505 Patients with Cervical Spondylotic Myelopathy. Spine (Phila Pa 1976) 46, 329-336, doi:10.1097/BRS.0000000000003783 (2021).

27 Fekete, T. F.et al. How does patient-rated outcome change over time following the surgical treatment of degenerative disorders of the thoracolumbar spine? Eur Spine $J$ 27, 700-708, doi:10.1007/s00586-0175358-2 (2018). 


\section{Figures}

796 patients were eligible and admitted to one of the participating institutions to undergo cervical spine surgery satisfied between April 2017 and October 2019.

153 patients were excluded due to surgery involving thoracic level and/or anterior surgery and/or fixation surgery.

151 patients were excluded due to the presence of ossification of posterior longitudinal ligament or yellow ligament.

492 patients were enrolled to our registry.

\begin{tabular}{|l|l|l|l|l|}
\hline $\begin{array}{l}\text { 24 patients did not undergo follow-up } \\
\text { one year after surgery. }\end{array}$ \\
$\begin{array}{l}10 \text { patients were excluded from the } \\
\text { analyses because of lack of answering }\end{array}$
\end{tabular}

\section{Figure 1}

Flowchart of the study population. 